\section{Paradigmas en el análisis de políticas públicas de salud: limitaciones y desafíos}

\author{
Walter Salas-Zapata, ${ }^{1}$ \\ Leonardo Ríos-Osorio, ${ }^{1}$ \\ Rubén Darío Gómez-Arias ${ }^{2}$ y \\ Xavier Álvarez-Del Castillo ${ }^{3}$
}

Forma de citar: Salas-Zapata W, Ríos-Osorio L, Gómez-Arias RB, Álvarez-Del Castillo X. Paradigmas en el análisis de políticas públicas de salud: limitaciones y desafíos. Rev Panam Salud Publica. 2012;32(1):77-81.

\begin{abstract}
RESUMEN
La investigación en politicas de salud se considera esencial para asegurar la efectividad y eficiencia de las políticas públicas. Los análisis de políticas públicas de salud (APPS) obedecen a diferentes propósitos; entre ellos, contribuir a resolver aquellos problemas que originaron la politica. Tal propósito enfrenta dos grandes obstáculos: 1) la polisemia y heterogeneidad de los modelos aplicados al análisis de políticas públicas, condiciones que dificultan la selección de los métodos de análisis y la valoración de sus alcances; y 2) los enfoques metodológicos tradicionales que limitan la capacidad de los análisis para contribuir a resolver los problemas detectados. En este ensayo se revisan los fundamentos epistemológicos de los modelos predominantes en APPS con el fin de valorar sus alcances y limitaciones. Se concluye que el desarrollo de nuevas perspectivas conceptuales podría mejorar la calidad de la investigación en politicas públicas y su capacidad para incidir favorablemente en las decisiones.
\end{abstract}

Palabras clave: políticas públicas de salud; gestión del conocimiento; investigación.

\footnotetext{
1 Universidad de Antioquia, Escuela de Microbiología, Medellín, Colombia. La correspondencia se debe enviar a Walter Salas-Zapata, wsalas@udea.edu.co

2 Universidad de Antioquia, Facultad Nacional de Salud Pública, Medellín, Colombia.

3 Universidad Politécnica de Cataluña, Cátedra Unesco de Sostenibilidad, Terrassa, España.
}

Las políticas públicas pueden entenderse como dispositivos para el control social generados en el contexto de la modernidad, que reflejan las interacciones de un sistema de actores y se expresan en sus reglas y modos de actuación, definiendo la forma de proceder frente a un asunto considerado de interés público; en el caso de la salud pública su asunto son los problemas que afectan la salud (1). El análisis de políticas públicas puede considerarse como una disciplina científica y como una ciencia social aplicada (2-4). Particularmente, se ha definido como un proceso multidisciplinar $(5,6)$ y multimetodológico (4) de gestión del conocimiento $(7,8)$ dirigido a explicar tanto las interacciones de influencia entre actores, instituciones, intereses e ideas (5) como la naturaleza y el resultado de las decisiones $\mathrm{y}$ acciones que emergen de tales interacciones.

Según los principios de las democracias liberales, las políticas públicas relacionadas con la salud deberían formularse para resolver un problema de salud considerado de interés público. En un sentido similar, los análisis de políticas públicas de salud (APPS) deberían también contribuir a la solución del problema destacando los aciertos y las limitaciones de las políticas y las intervenciones basadas en ellas. No obstante, la heterogeneidad de tradiciones ideológicas, conceptuales y metodológicas utilizadas por los APPS $(8,9)$ ha generado un marco polisémico y confuso que dificulta su evaluación. Esta dificultad se refleja en las deficiencias metodológicas de las investigaciones publicadas en los últimos años $(5,10)$.

Este ensayo pretende revisar los fundamentos epistemológicos de los modelos predominantes en APPS desde mediados del siglo xx, examinando sus alcances y limitaciones, con el fin de proponer opciones que mejoren la utilidad y la capacidad de los estudios para contribuir a la solución de los problemas de salud.

\section{LOS ENFOQUES EN EL ANÁLISIS DE POLÍTICAS PÚBLICAS}

Los análisis de políticas públicas albergan una amplia variedad de enfoques que, desde la perspectiva epistemológica, podrían clasificarse en tres categorías: positivistas, sociohistóricos y pospositivistas (cuadro 1).

Los enfoques positivistas consideran a las políticas públicas como procesos racionales que incorporan datos y evidencia "objetiva" para predecir cursos de acción y tomar mejores decisiones $(3,6)$. Ejemplo de estos son los análisis de toma de decisiones, de costobeneficio y de costo-efectividad (7).

Los enfoques sociohistóricos asumen las políticas públicas como procesos resultantes de las interac- 
CUADRO 1. Enfoques en el análisis de políticas públicas y algunos autores de referencia

\begin{tabular}{cll}
\hline \multicolumn{1}{c}{ Enfoque } & \multicolumn{1}{c}{ Paradigma o tipo de análisis } & \multicolumn{1}{c}{ Autor (referencia) } \\
\hline Positivista & Normativo-prescriptivo, sinóptico, empírico, analítico & Pérez (7) \\
& "Conocimiento en" la política & Lasswell (11) \\
& "Análisis de" la política & Parsons (12) \\
& Hiperracional & Cruz y Petrizzo (13) \\
& Tecnocrático, positivista lógico & Torgerson (8) \\
Sociohistórico & Conductista & Garsón (14) \\
& Positivo, antisinóptico, incrementalista, pluralista & Pérez (7) \\
& "Conocimiento de" la política & Lasswell (11) \\
& "Análisis de" la política & Parsons (12) \\
& "Hiperpolitizado" & Cruz y Petrizzo (13) \\
Pospositivista/crítico & Contextualizador & Torgerson (8) \\
& Neopluralista, humanista & Garsón (14) \\
& Pospositivista/crítico & Torgerson (8) \\
& Constructivismo & Cruz y Petrizzo (13) \\
& Teoría de sistemas & Roth (9), Fafard (15) \\
\end{tabular}

ciones entre grupos de interés; analizan los juicios de valor, los grupos de interés y los contextos sociohistóricos de las acciones y decisiones políticas para entender cómo y por qué ciertos asuntos públicos se incluyen en la agenda de gobierno mientras otros se excluyen; y cómo y por qué se eligen unos u otros cursos de acción.

Los enfoques pospositivistas critican los enfoques positivistas y sociohistóricos porque la distancia que toman los investigadores frente a los objetos de estudio les impide comprender la realidad en su complejidad y comprometerse con la solución de los problemas que las políticas públicas pretenden resolver. Entre ellos predomina el enfoque crítico. Este sugiere que tanto las políticas públicas, como el investigador y el problema que la política pretende resolver forman parte del mismo orden social. En consecuencia, para entender una política pública es necesario comprender el orden social que la enmarca.

\section{Origen de los enfoques en el análisis de políticas públicas}

Los enfoques de análisis de políticas públicas responden a condiciones sociohistóricas, epistemológicas y teóricas particulares (figura 1). A principios del siglo XX no se hablaba de políticas públicas debido, en parte, al predominio de los principios liberales occidentales que propendían por Estados no interventores. La crisis económica de la posguerra obligó a las democracias liberales a adoptar modelos intervencionistas que desarrollaron las políticas públicas como dispositivos de control. Por la misma época el positivismo promovido por el Círculo de Viena se fortaleció como paradigma científico. En este contexto, Lasswell impulsó el estudio de las políticas públicas desde dos enfoques: el "conocimiento en" la política, interesado por los métodos y habilidades para la toma de decisiones, y el "conocimiento de" las políticas, comprometido con la democracia y la resolución de problemas sociales $(8,11,14)$. El primero, esencialmente positivista, fue fortalecido por los Esta- dos intervencionistas que promovieron la formación positivista y conductista de profesionales vinculados a las políticas de gobierno $(2,7,14)$. El segundo, predominantemente sociohistórico, se fortaleció con las ideas de Kuhn sobre la importancia del contexto en la generación de conocimiento científico (17) y cuando las prescripciones positivistas mostraron sus limitaciones para transformar la realidad (14).

Ambos enfoques mostraron sus deficiencias para explicar la dinámica de los problemas de interés público y de las políticas públicas; adicionalmente, los resultados prometidos por los investigadores no se correspondían con los resultados reales (7). El desencanto frente a estos resultados dio lugar a los enfoques pospositivistas en el APPS, que propendían por un vínculo más estrecho entre el investigador y el problema de investigación, y por un compromiso real del estudio con la solución (8).

\section{ASPECTOS CRÍTICOS EN EL ANÁLISIS DE POLÍTICAS PÚBLICAS DE SALUD}

Los cambios económicos, políticos y sociales de finales del siglo $X X$ transformaron la concepción y gestión de las políticas públicas. En esencia, estas siguieron considerándose como decisiones y acciones emanadas de relaciones de poder, pero ahora se reconoce que obedecen a relaciones interorganizacionales y transfronterizas (5) las cuales imponen a las políticas públicas un carácter dinámico, impredecible y de difícil control; estas condiciones evidencian ciertas limitaciones de los APPS relacionadas con: 1) el enfoque y el método, 2) la consideración de actores y contextos, 3) la utilización de teorías y 4) la relación entre investigadores y actores.

\section{El enfoque y el método empleados en los análisis de políticas públicas}

Aunque no se ha hecho una revisión sistemática del tema, la bibliografía publicada sugiere un predominio de los enfoques positivistas en los análisis de 
FIGURA 1. Desarrollo de enfoques de análisis de políticas públicas en el siglo XX

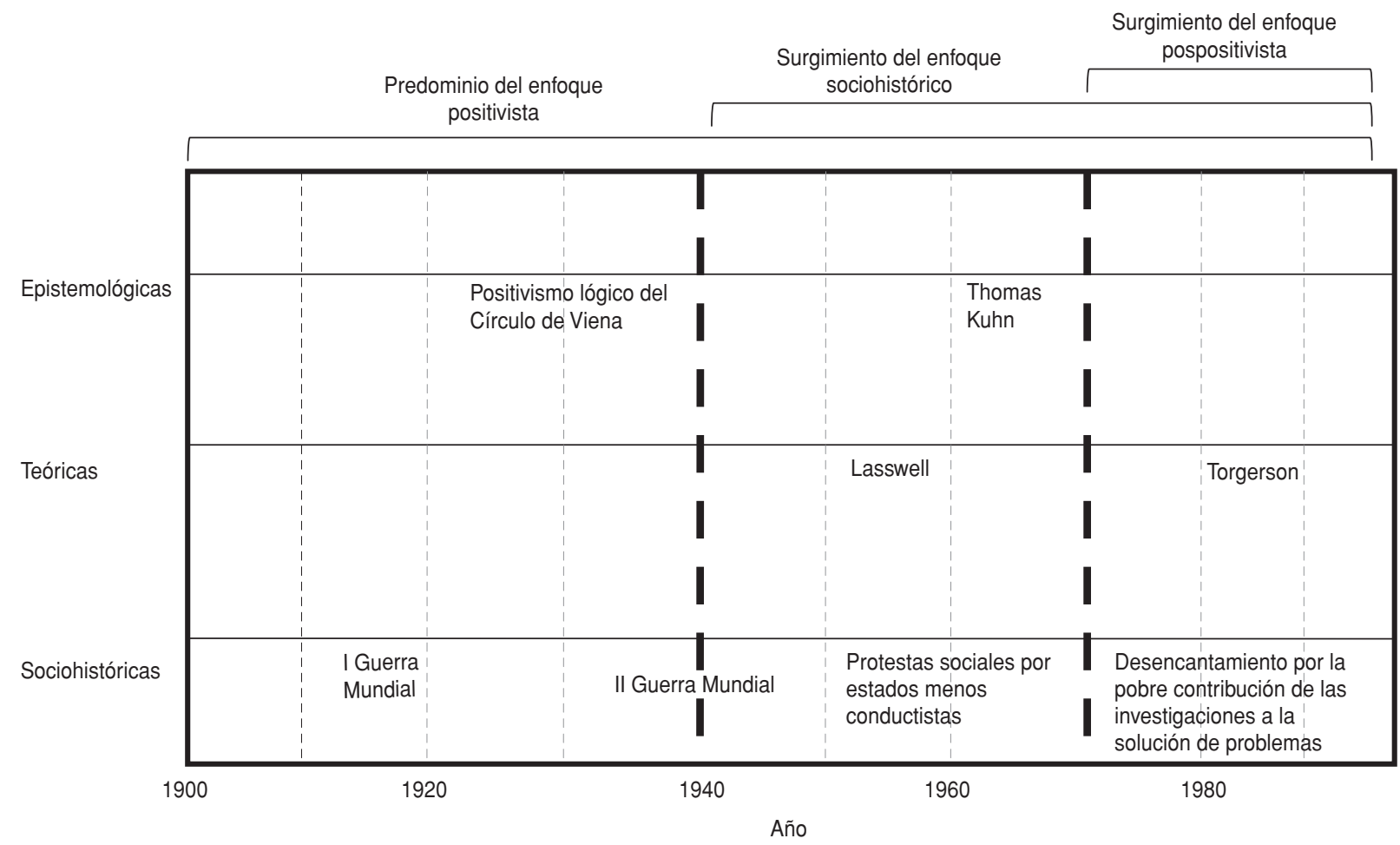

políticas públicas $(5,15,18)$ destacándose los análisis de proceso (stage model), de costo-beneficio y de costo-efectividad (19). Los enfoques cualitativos son menos utilizados (10). De 27747 artículos indexados en Medline al 14 de julio de 2011 bajo el descriptor health policy analysis, $42,6 \%$ se refería a los costos y solo $3,4 \%$ a politics. Los APPS de corte positivista suelen centrarse en elementos medibles y verificables, y excluyen categorías esenciales que no son observables empíricamente, como los juicios de valor, los intereses y los contextos $(5,15)$; en tal sentido, su capacidad para contribuir a la solución de los problemas de salud podría ser muy limitada (20).

\section{Consideración de actores y contextos}

Pocos APPS detallan las experiencias de los actores durante la implementación de las políticas públicas y las influencias que ejercen sobre ellos las estructuras burocráticas o los contextos social, político y cultural $(5,10,21)$. Esta omisión impide a los APPS dar cuenta de las consecuencias no previstas de las políticas públicas, de los dispositivos de control y de las relaciones de poder; adicionalmente, limita la utilidad de sus resultados porque no revela los contextos particulares de aplicabilidad del conocimiento. Los análisis sociohistóricos y pospositivistas críticos permiten hacer estos abordajes, pero desconocen el carácter económico y ecológico del entorno, cuya influencia ha sido identificada en casos como las políticas de control del paludismo $(22,23)$.

\section{Utilización de teorías y rigor metodológico}

Los APPS han sido criticados por omitir la intencionalidad del análisis y los fundamentos teóricos que sustentan los hallazgos $(5,10,24)$. En el caso de los análisis de reformas sanitarias, los investigadores han señalado problemas para identificar las preguntas de investigación y los criterios de selección de los métodos, para comunicar los resultados y para aprovechar las bases de datos (19). Estas debilidades metodológicas hacen más difícil valorar el alcance de sus resultados, limitan su transferibilidad a contextos reales y sugieren que prescindir de fundamentos conceptuales firmes reduce la capacidad de los APPS para contribuir a la solución del problema de salud (25).

\section{Relación entre investigadores y decisión}

Las publicaciones sugieren que la investigación realizada en materia de políticas públicas de salud desempeña un papel secundario en el desarrollo de las mismas porque: 1) con frecuencia las decisiones se toman con base en criterios diferentes al conocimiento derivado de las investigaciones $(15,19,20,26)$; 2) los intereses de los investigadores no siempre coinciden con los intereses de los tomadores de decisiones, quienes prestan poca atención a los hallazgos; 3) los investigadores y hacedores de políticas "rara vez obran de manera racional con arreglo a fines, pues lo hacen desde su historia emocional, sus aspiraciones personales, sus lealtades de grupo, las rutinas aprendidas de su 
entorno y su posición en el grupo" (19); 4) los hacedores de políticas tienen dificultades para comprender las investigaciones y confían poco en ellas; 5) los investigadores subestiman a los tomadores de decisiones y los procesos políticos; y 6) los contextos socioeconómicos, culturales y ambientales y epidemiológicos son muy heterogéneos y limitan las extrapolaciones.

El éxito de esta interacción se ha relacionado, en cambio, con la comunicación entre investigadores y hacedores de políticas, tanto durante el diseño e interpretación de los estudios como durante formulación e implementación de las políticas $(19,25)$.

\section{DESARROLLO DE NUEVOS ENFOQUES EN EL ANÁLISIS DE POLÍTICAS PƯBLICAS DE SALUD}

Para superar estas limitaciones y desarrollar enfoques innovadores, los APPS podrían enfatizar los siguientes aspectos del análisis.

\section{Abordaje integral del objeto de estudio}

Las relaciones de poder, la correlación de fuerzas, las alianzas, los intereses y los conflictos son condiciones inherentes a las políticas públicas (6) y el entorno tiene un carácter social, ecológico y económico, que determina su naturaleza y su dinámica. En consecuencia, los enfoques del análisis deberían dar cuenta de estos aspectos en su mutua determinación; así mismo, deberían considerar las políticas públicas de salud como dispositivos de control al servicio de intereses específicos de los involucrados que varían según sus contextos históricos.

\section{Diseño y utilización de métodos de investigación relacionales}

Dado que el alcance de los APPS depende en gran manera de la comunicación entre investigadores y actores sociales, se deberían utilizar métodos de investigación que posibiliten dicha interacción, en tres sentidos: 1) generar un espacio de aprendizaje social. Una investigación no contribuirá a transformar la realidad mientras no modifique la estructura cognitiva y los comportamientos de los involucrados. Este aprendizaje no depende solo de generar nueva información sino del escenario en el que los actores sociales se enfrentan a un conocimiento que se reconstruye con la experiencia; 2) producir conocimiento relacional. Si los métodos promueven una relación activa entre investigadores y actores sociales, el conocimiento generado puede reflejar mejor la dinámica de las políticas públicas; y 3) reconocer al investigador como actor comprometido con la solución del problema y en tal sentido como actor político, sin perjuicio de la objetividad que se deriva de la triangulación y de la rigurosidad de los métodos.

\section{CONCLUSIONES}

Los APPS realizados en los últimos años se han amparado predominantemente en enfoques positivistas, y minoritariamente en enfoques sociohistóricos y pospositivistas. Los enfoques positivistas suelen desconocer procesos subjetivos y contextuales que son esenciales en una política pública, y su predominio como paradigma hegemónico podría limitar el alcance de los APPS. Aunque menos utilizados, los enfoques sociohistóricos podrían contribuir a resolver estas lagunas; sin embargo, no aseguran un compromiso del investigador con el problema ni una adecuada interacción con los tomadores de decisiones que garantice la aplicación del conocimiento.

Los investigadores podrían mejorar la utilidad de los APPS para resolver los problemas que son objeto de las políticas si definen adecuadamente modelos que consideren, entre otros, los siguientes argumentos: las políticas públicas son la expresión de relaciones de poder, que actúan como dispositivos de control; el entorno social, económico y ecológico es un determinante de toda política pública de salud $\mathrm{y}$, como tal, es un componente esencial de los APPS; los modelos y métodos deberían constituirse como espacios de gestión del conocimiento que faciliten el aprendizaje, la participación y la interacción de los investigadores y los demás actores sociales para facilitar la comunicación y la cooperación entre ellos.

\section{ABSTRACT}

\section{Paradigms in the analysis of public health policies: limitations and challenges}

Research on health policies is considered essential to ensure the effectiveness and efficiency of public policies. Analyses of public health policies have various objectives, including helping to solve the problems for which the policy was originated. That objective faces two large obstacles: (1) the ambiguity and heterogeneity of the models applied for the analysis of public policies, conditions that hinder the selection of analytical methods and the assessment of the scope of the objective; and (2) the traditional methodological approaches that limit the capacity of analyses to help solve the problems detected. This paper reviews the epistemology of the predominant models of public health policy analysis in order to assess their scope and limitations. It concludes that the development of new conceptual approaches could improve the quality of research on public policies and their ability to favorably impact decisions.

Key words: health public policy; knowledge management; research. 


\section{REFERENCIAS}

1. Gómez-Arias R. Atención primaria de salud y políticas públicas. Rev Fac Nac Salud Publica. 2011;28(3):283-93.

2. Lasswell H. La orientación hacia las políticas [1951]. En: Aguilar L (ed.). El estudio de las políticas públicas. $3^{\mathrm{a}}$ ed. México D.F.: Editorial Miguel Ángel Porrúa; 2003. Pp. 79-104.

3. Landau M. El ámbito propio del análisis de políticas. En: Aguilar L (ed.). El estudio de las políticas públicas. $3^{\text {a }}$ ed. México D.F.: Editorial Miguel Ángel Porrúa; 2003. P. 278.

4. Meny I, Thoenig J. Las políticas públicas. Barcelona: Ariel; 1992.

5. Walt G, Shiffman J, Schneider $H$, Murray S, Brugha R, Gilson L. 'Doing' health policy analysis: methodological and conceptual reflections and challenges. Health Policy Plann. 2008;23(5): 308-17.

6. Gilson L. Health policy and systems research. A methodology reader. Geneve: World Health Organization; 2012.

7. Pérez M. Análisis de políticas públicas. Granada: Universidad de Granada; 2005.

8. Torgerson D. Entre el conocimiento y la política: tres caras del análisis de política [1986]. En: Aguilar L (ed.). El estudio de las políticas. México D.F.: Editorial Miguel Ángel Porrúa; 2003. Pp. 197-238.

9. Roth A. Enfoques y teorías en el análisis de políticas públicas, cambio de la acción pública y transformación del Estado. En: Cuervo J, Salazar C, Jolly J, Tournier C, Roth A, Velez G (eds.). Ensayos sobre políticas públicas. Bogotá: Universidad Externado de Colombia; 2007. Pp. 28-63.

10. Gilson L, Raphaely N. The terrain of health policy analysis in low and middle income countries: a review of published literature 1994-2007. Health Policy Plann. 2008;23(5):294-307.

11. Lasswell H. La concepción emergente de las ciencias de políticas [1971]. En: Aguilar L (ed.). El estudio de las políticas públicas. $3^{a}$ ed.: Editorial Miguel Ángel Porrúa; 2003. Pp. 105-18.

12. Parsons $W$, Acevedo A, Méndez $H$, Lendo T. Políticas Públicas. Una introducción a la teoría y la práctica del análisis de políticas públicas. México D.F.: Flacso; 2007.

13. Cruz C, Petrizzo M. El estudio de las políticas públicas: el estado de la disciplina y la consolidación democrática en América Latina; 2008. Disponible en: http:/ / petrizzo.googlepages.com/Politicas Publicas.pdf Acceso el 25 de julio de 2011.

14. Garsón D. De la ciencia de políticas al análisis de políticas: veinticinco años de progreso. En: Aguilar L (ed.). El estudio de las políticas. México D.F.: Editorial Miguel Ángel Porrúa; 2003. Pp. 149-80.

15. Fafard P. Evidence and Healthy Public Policy: Insights from Health and Political Sciences. Quebec: National Collaborating Centre for Healthy Public Policy; 2008.

16. Eslava J, Puente C. Análisis de las políticas públicas: una aproximación desde la teoría de los sistemas sociales. Bogotá: Cendex; 2003. P. 37.

17. Kuhn T. The Structure of Scientific Revolutions. Chicago: University of Chicago Press; 1970.

18. Molina G, Roth A. Introducción. En: Molina G, Cabrera G (eds.). Políticas públicas en salud: aproximación a un análisis. Colombia: Universidad de Antioquia; 2008. Pp. 3-6.

19. Gómez R, Orozco D, Rodríguez F, Velasquez W. Políticas públicas en salud: relación entre investigación y decisión. Rev Fac Nac Salud Publica. 2006;24(2):105-18.

20. Hertin J, Turnpenny J, Jordan A, Nilsson M, Russel D, Nykvist B. Rationalising the policy mess? Ex ante policy assessment and the utilisation of knowledge in the policy process. Environ Plann. 2009;41(5):1185-200.

21. Stein E, Tommasi M, Echebarría K, Lora E, Payne M. La política de las políticas públicas. En: BID (ed.). Cambridge: David Rockefeller Center for Latin American Studies-Harvard University; 2006.

22. Carmona-Fonseca J. La malaria en Colombia, Antioquia y las zonas de Urabá y Bajo Cauca: panorama para interpretar la falla terapéutica antimalárica. Parte I. Iatreia. 2003;16(4):299-318.

23. Pattanayak S, Dickinson K, Corey C, Murray B, Sills E, Kramer R. Deforestation, malaria, and poverty: a call for transdisciplinary research to support the design of cross-sectoral policies. Sustain Sci Prac Policy. 2006;2(2):45-56.

24. Cheung K, Mirzaei M, Leeder S. Health policy analysis: a tool to evaluate in policy documents the alignment between policy statements and intended outcomes. Aust Health Rev. 2010;34:405-13.

25. Bronfman M, Langer A, Trostle J. De la investigación en salud a la política. La difícil traducción. México D.F.: Manual Moderno; 2000.

26. Almeida C, Bascolo E. Use of research results in policy decision-making, formulation, and implementation: a review of the literature. Cad Saude Publica. 2006;22(S7-S33).

Manuscrito recibido el 13 de octubre de 2011. Aceptado para publicación, tras revisión, el 11 de abril de 2012. 